\title{
Body Segmentation using Gradient Background and Intra-Frame Collision Responses for Markerless Camera-Based Games
}

\author{
Jun-Geon Kim* and Daeho Lee ${ }^{\dagger}$
}

\begin{abstract}
We propose a novel framework for markerless camera-based games. By using a visual camera, our method may yield robust human body segmentation with high performance comparable to the segmentation using depth cameras. The edges of human bodies are detected by subtracting gradient backgrounds, and human body regions are segmented by the operations based on mathematical morphology. Collisions between detected regions and virtual objects are determined by finding the colliding time using intra-frame positions of virtual objects. Experimental results show that the proposed method may produce robust segmentation of human bodies, thereby and the collision responses are more accurate than previous methods. Therefore, the proposed framework can be widely used in camera-based games requiring high performance.
\end{abstract}

Keywords: Body segmentation, Gradient background, Mathematical morphology, Collision response, Camera-based games

\section{Introduction}

Human activity analysis has been a challengeable issue of computer vision research [1]. The capability of analyzing human activities has led to the development of various applications such as surveillance systems, remote control systems and computer games [2-14]. Especially, human movements are used as their gestures in camera-based games.

Human body segmentation is a classically difficult topic $[9,10]$. To segment human bodies, background subtraction is commonly used [2], where the pixel intensities of backgrounds are modeled with a Mixture of Gaussian $(\mathrm{MoG})$ distribution, and they are recursively updated [15, 16]. Unfortunately, intensity backgrounds are sensitive to small intensity variations. Color segmentation $[11,17]$ may be an alternative method for human detection, however only skin or particular color regions can be detected using this method. Also color variation may result in poor segmentation. To improve the performance of movement detection, motion captures (e.g., data gloves) or special markers may be used [18-20]. These cumbersome captures or markers are, however, always necessary to play the games.

In recent years, human activity analysis using depth cameras $[21,22]$, has been widely issued [24-26]. The depth information is retrieved using the time-of-flight (TOF) principle [23] or structured lighting. As shown in Fig. 1, the use of depth cameras may yield accurate segmentation results, compared to the visual cameras;

$\dagger$ Corresponding Author: Humanitas College, Kyung Hee University, Yongin-si, Gyeonggi-do, Korea. (nize@khu.ac.kr)

* Department of Electronics and Radio Engineering, Kyung Hee

University, Yongin-si, Gyeonggi-do, Korea. (joongoen@khu.ac.kr)

Received February 28, 2015; Accepted September 30, 2015

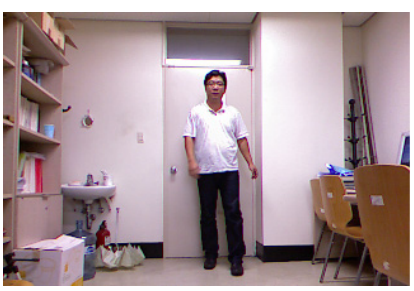

(a)

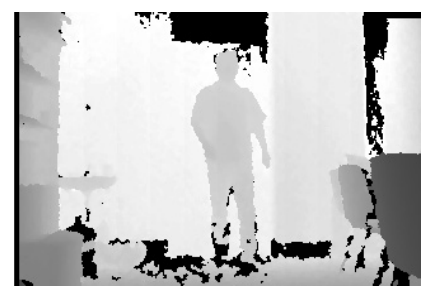

(b)
Fig. 1. Visual image (a) and its depth image (b).

however, an expensive depth camera is always necessary and they work only in limited regions.

This paper is aimed at developing a novel framework for markerless camera-based games by human body segmentation having high performance comparable to the depth cameras. To achieve this aim, we apply a novel background subtraction method using gradient and an intra-frame collision response. The proposed framework can be used adequately to markerless camera-based games with accurate collision responses.

\section{Organization of Proposed Framework}

The proposed framework uses only a visual camera connected to a PC as shown in Fig. 2(a), where the background of playing regions is not severely cluttered. For human interacting with virtual objects in the game screen of Fig. 2(a), first of all, the human bodies should be segmented in every frame. This procedure is performed by gradient background subtraction, which is composed of several steps including gradient map calculation of a frame, background subtraction, and morphological operations. The detail explanation on the process appears in section 3. 

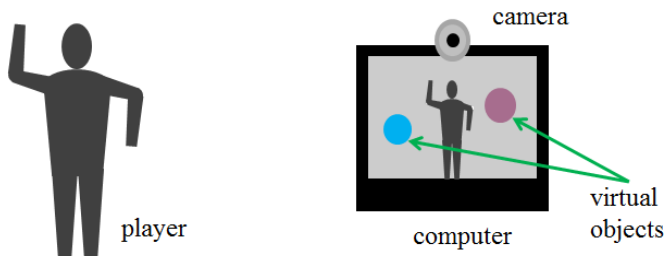

(a) Game environment

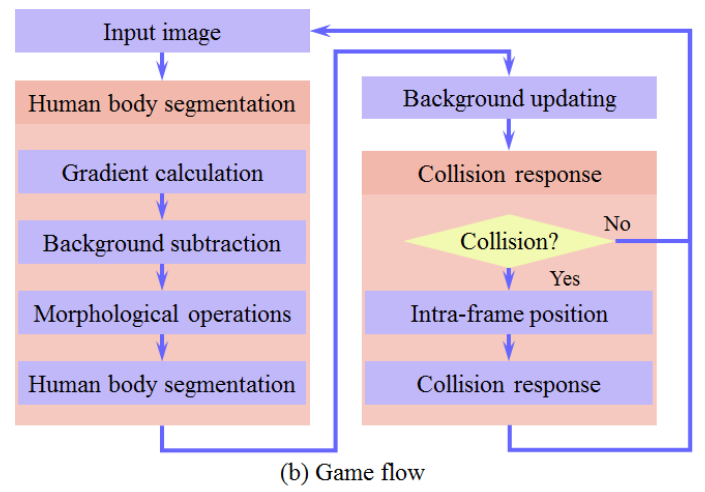

Fig. 2. The proposed framework.

Virtual objects may be generated by the game scenario and each piece moves with its velocity. When the virtual objects collide with segmented human body region in computer screen, the objects respond by intra-frame collision responses which is in detail depicted in section 5 . Fig. 2(b) shows the entire logical flow of the proposed framework.

\section{Gradient Background Subtraction}

Gradients of an image are simply calculated by gradient operators such as Sobel, Roberts cross and Prewitt operators. In this paper, we use the Sobel operator because of its capability of noise suppression. To detect edges, the gradient magnitude is commonly used, but we use a gradient vector $\nabla I$, so the gradient background $\mathbf{B}$ is a $2 \mathrm{D}$ array of vectors. A gradient vector of a gray image $I_{t}$ at time $t$ is calculated by

$$
\begin{gathered}
\nabla I_{t}(x, y)=\left(\frac{\partial I_{t}(x, y)}{\partial x}, \frac{\partial I_{t}(x, y)}{\partial y}\right)=\left(G_{x, t}, G_{y, t}\right) \\
=\left(I_{t}(x+1, y+1)+2 I_{t}(x+1, y)+I_{t}(x+1, y-1)\right. \\
-I_{t}(x-1, y+1)-2 I_{t}(x-1, y)-I_{t}(x-1, y-1), \\
I_{t}(x+1, y+1)+2 I_{t}(x, y+1)+I_{t}(x-1, y+1) \\
\left.-I_{t}(x+1, y-1)-2 I_{t}(x, y-1)-I_{t}(x-1, y-1)\right)
\end{gathered}
$$

At the first frame, the gradient background $\mathbf{B}_{0}$ is $\nabla I_{0}$, and the background is updated over time as follows:

$$
\begin{aligned}
& B_{x, t}=B_{x, t-1}+\alpha\left(1-H_{t-1}\right)\left(G_{x, t-1}-B_{x, t-1}\right) \\
& B_{y, t}=B_{y, t-1}+\alpha\left(1-H_{t-1}\right)\left(G_{y, t-1}-B_{y, t-1}\right)
\end{aligned}
$$
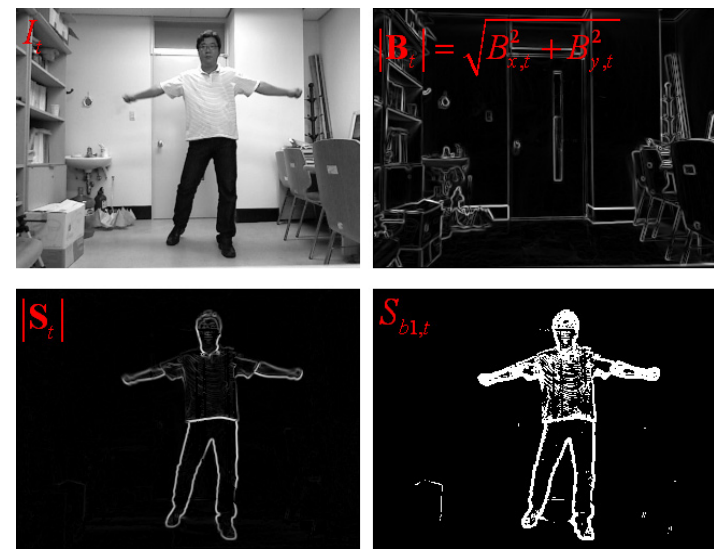

Fig. 3. An example of gradient background subtraction.

where $H_{t-1}$ denotes a binary human mask at time $t-1$ and is calculated after human body segmentation and $\alpha$ denotes the updating gain.

To detect evidences of human bodies, the gradient at the current frame is subtracted by the gradient background as follows:

$$
\mathbf{S}_{t}=\nabla I_{t}-\mathbf{B}_{t}
$$

To determine the edges of human bodies, $\mathbf{S}_{t}$ is binarized to $S_{b 1, t}$ as follows:

$$
S_{b 1, t}(x, y)=\left\{\begin{array}{cc}
1 & \text { if }\left|\mathbf{S}_{t}(x, y)\right|>\tau \wedge\left|\nabla I_{t}\right|>\left|\mathbf{B}_{t}\right| \\
0 & \text { otherwise }
\end{array}\right.
$$

where $\tau$ denotes a threshold value. The second condition of (4) is very important. If this condition is omitted, false estimated background pixels cannot be corrected, because we use the binary human mask $H$ in updating the background by (2). Fig. 3 shows an example of gradient background subtraction.

\section{Human Body Segmentation}

To detect human body regions, closed edges must be filled; however, $S_{b 1, t}$ has many unlinked edges. Thus, we apply some operations based on mathematical morphology as shown in Fig. 4. We first apply dilation to $S_{b 1, t}$ as follows:

$$
S_{b 2, t}=S_{b 1, t} \oplus A_{1}
$$

where $\oplus$ denotes the dilation operator, and $A_{1}$ is a structuring element shaped by a $n \times m$ cross.

Before region filling $S_{b 2, t}$, small connected regions are removed using connected component analysis; $S_{b 3, t}$ is the binary image after removing small regions. And $S_{b 4, t}$ is the binary image after filling $S_{b 3, t}$. Because $S_{b 4, t}$ is 
dilated, we apply erosion to $S_{b 4, t}$ as follows:

$$
S_{b 5, t}=S_{b 4, t} \ominus A_{1}
$$

where $\ominus$ denotes the erosion operator. Finally, an opening operation is applied to $S_{b 5, t}$ in order to split non body regions as follows:

$$
S_{b 6, t}=\left(S_{b 5, t} \ominus A_{2}\right) \oplus A_{2},
$$

where $A_{2}$ is a structuring element shaped by an $l \times l$ square. By the morphological operations, human body regions $S_{b 6, t}$ are segmented and this mask is used as $H$ in the background estimation of (2).

\section{Collision Detection and Response}

To detect and respond between human bodies and virtual objects, we calculate the boundaries of $S_{b 6, t}$ and the boundaries are smoothed by a Gaussian kernel as shown in the last image of Fig. 4.

In $[10,13]$ and [14], collisions are detected by an overlapped ratio, and collision responses are estimated
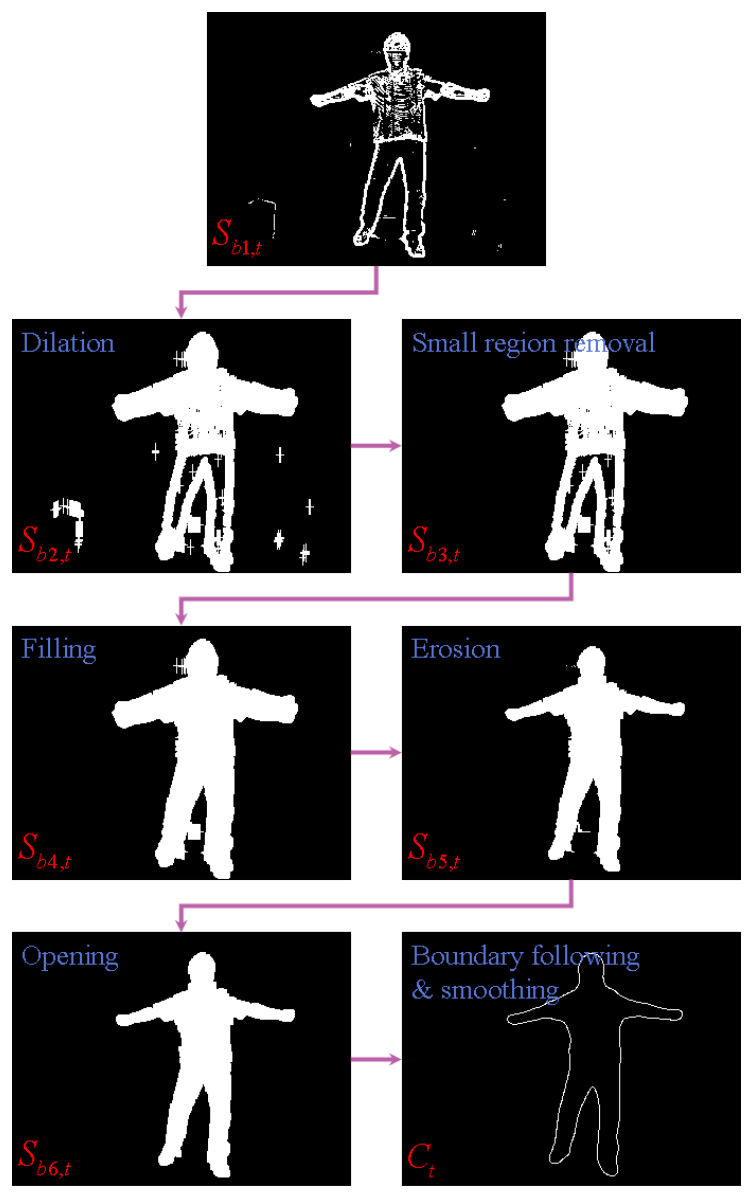

Fig. 4. An example of human body segmentation. using collision planes. However, these methods do not consider intra-frame positions as shown in Fig. 5; that is, the positions of virtual objects only at discrete frame times are considered.

In this paper, we also use the overlapped ratios for collision detections, but intra-frame positions are considered for collision responses as shown in Fig. 6. To find the precise colliding time, we shift the positions of virtual objects at intervals of $-\Delta(0<\Delta<1)$ from time $t$, and find the maximum $n \Delta$ colliding with bodies, where the colliding time is $t-n \Delta$. When the overlapped radio of the collision region between bodies and a virtual object is more than 0 , the virtual object is determined as a collided object, and then the colliding time $t-n \Delta$ is found. The motion vector of a collided object at time $t+1$ is calculated by

$$
\mathbf{v}_{t+1}=-2\left(\mathbf{v}_{t} \cdot \mathbf{n}_{t-n \Delta}\right) \mathbf{n}_{t-n \Delta}+\mathbf{v}_{t}
$$

where $\mathbf{v}_{t+1}$ and $\mathbf{v}_{t}$ are the motion vectors at time $t+1$ and $t$, respectively, and $\mathbf{n}_{t-n \Delta}$ is the normal vector of the colliding plane estimated as the vector starting the centroid of the collision region to the center of the collided object. Finally, the position $(x, y)$ of the collided object at time $t+1$ is calculated by

$$
\begin{aligned}
& x_{t+1}=x_{t-n \Delta}+v_{t+1}^{x}(1+n \Delta) \\
& y_{t+1}=y_{t-n \Delta}+v_{t+1}^{y}(1+n \Delta)
\end{aligned},
$$

where $v_{t+1}^{x}$ and $v_{t+1}^{y}$ are the $x$ and $y$ components of $\mathbf{v}_{t+1}$.

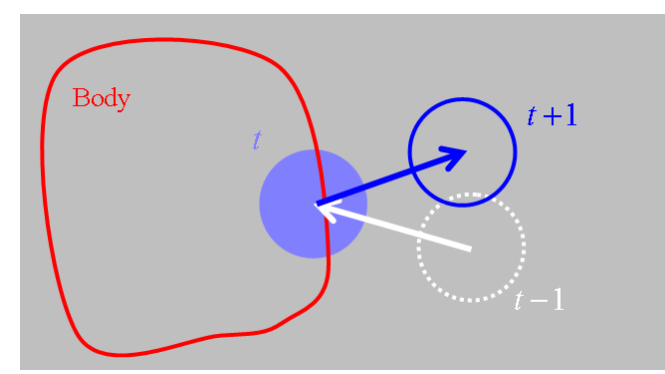

Fig. 5. Collision response of [6], [9] and [10].

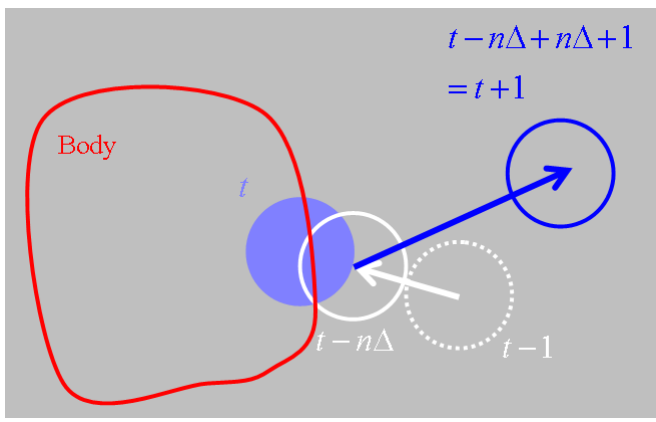

Fig. 6. The proposed collision response method. 


\section{Experimental Results}

The proposed method was tested on a Pentium PC (Core ${ }^{\mathrm{TM}} 2$ Duo, $\left.2.40 \mathrm{GHz}\right)$. The test images were acquired from a camera with the duration of $33 \mathrm{~ms}$. The resolution of the test image is $320 \times 240$ ( 8 bit grayscale).

In the experiments, we used $\tau=45$ for the background subtraction, a $7 \times 15$ cross as the structuring element for the dilation and erosion operations of $S_{b 2, t}$ and $S_{b 5, t}$, and a $5 \times 5$ square for the opening operation of $S_{b 6, t}$. When different cameras are used, these parameters may be adjusted by various threshold methods and image resolution. Human bodies were correctly segmented in various environments as shown in Fig. 7, and Fig. 8 shows the detection of the body according to the lighting conditions, while false segmentations were rarely observed as shown in Fig. 9.

The false segmentations were not consecutively detected for several frames, so these are acceptable for games. In addition, players almost could not notice these errors because the games are played very quickly at 30 frames/ second. To evaluate the performance of the proposed background updating, we tested an image sequence starting from a frame image in which a player exists as shown in Fig. 10. After 60th frame, human bodies were correctly segmented, and gradient backgrounds were correctly updated after 150th frame. As a result, the background can be stably estimated after 5 seconds when a player dynamically moves.

To compare our detection method with the methods using intensity backgrounds, we generated the background images updated by MoG as shown in Fig. 11. In game
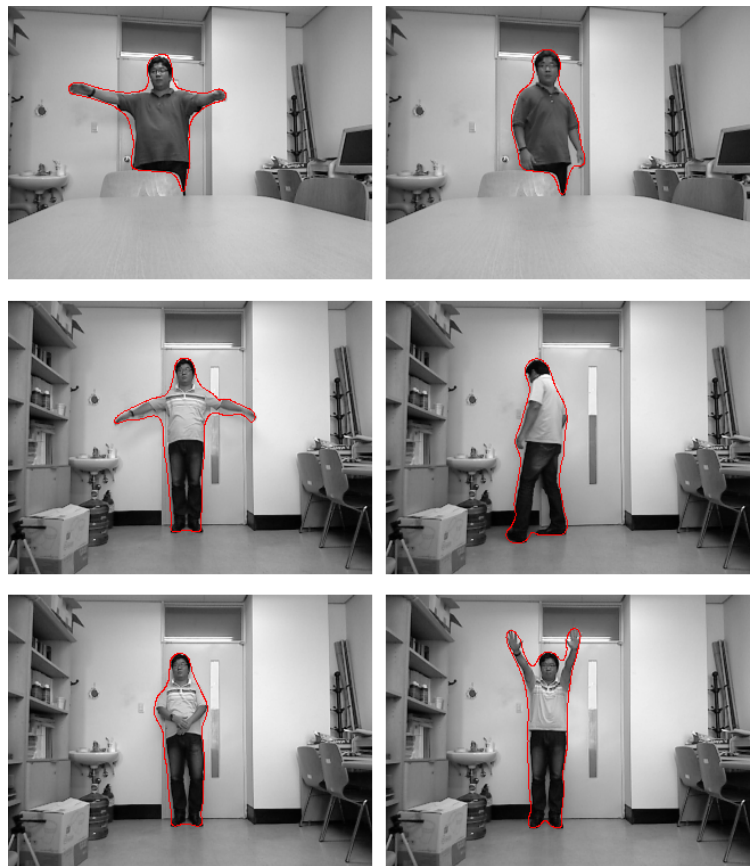

Fig. 7. Results of human bodies (1).
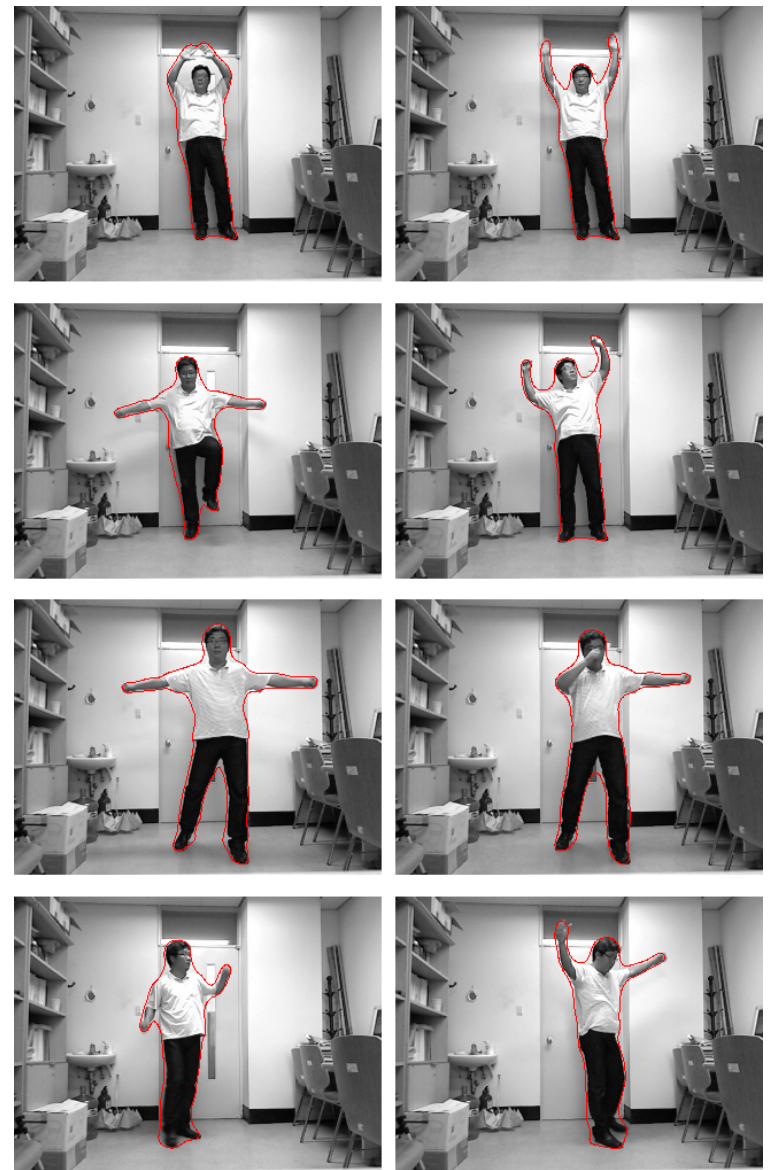

Fig. 8. Results of human bodies (2).
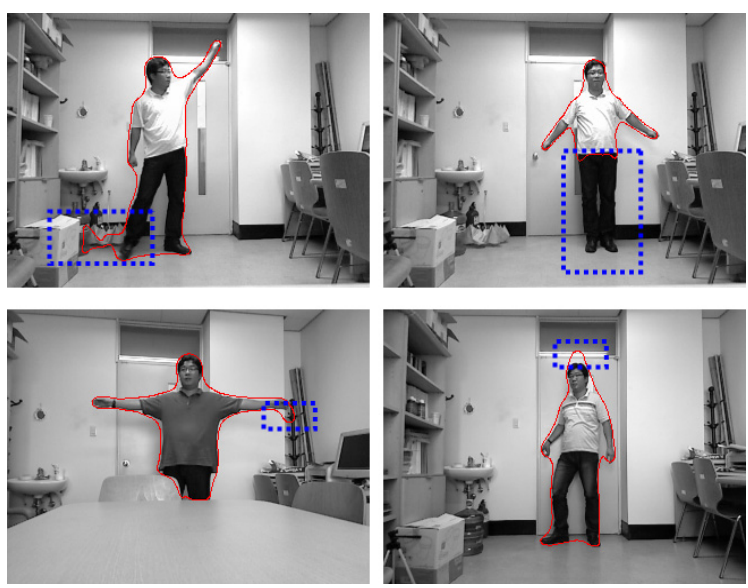

Fig. 9. Examples of false segmentations.

environments, players act in the limited space, so many foreground regions should be exposed in the backgrounds updated by MoG. In addition, static background cannot be used for body segmentation, because the segmentation results are very sensitive to luminance changing and initial manual setup processing is needed. So, the false segmentations gradually increase as time goes by as shown in Fig. 12. In the results segmented by low threshold levels, 
the regions having similar value with background pixels are not segmented, while false positive regions increase in the results with high threshold levels.
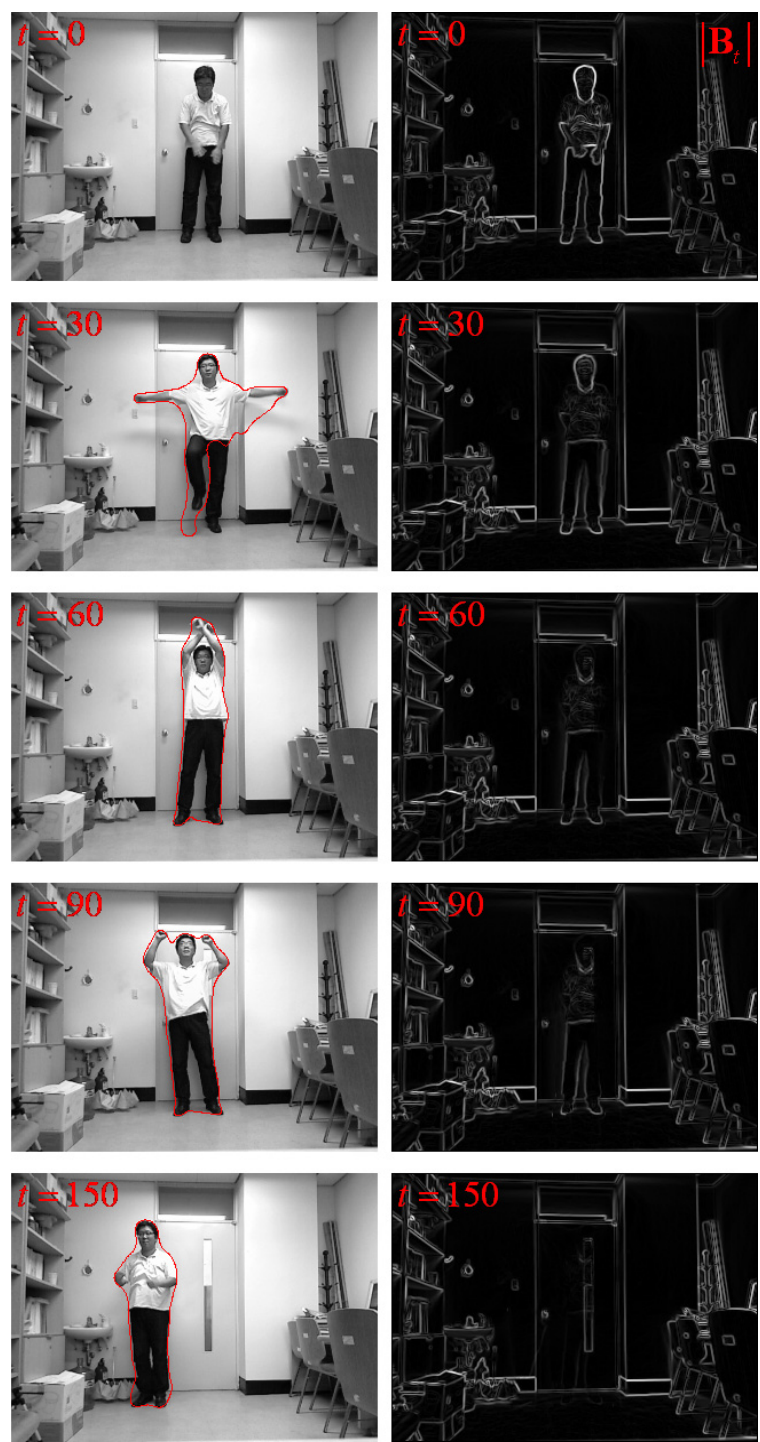

Fig. 10. Human body detection and background estimation.
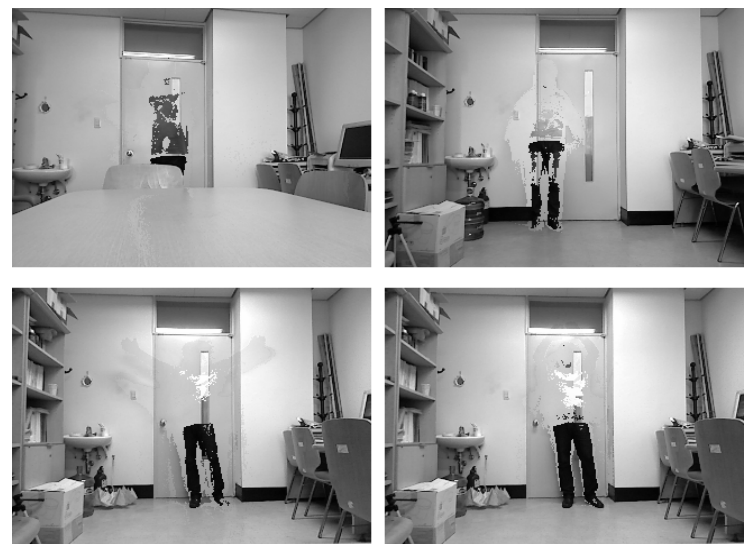

Fig. 11. Background images updated by MoG.
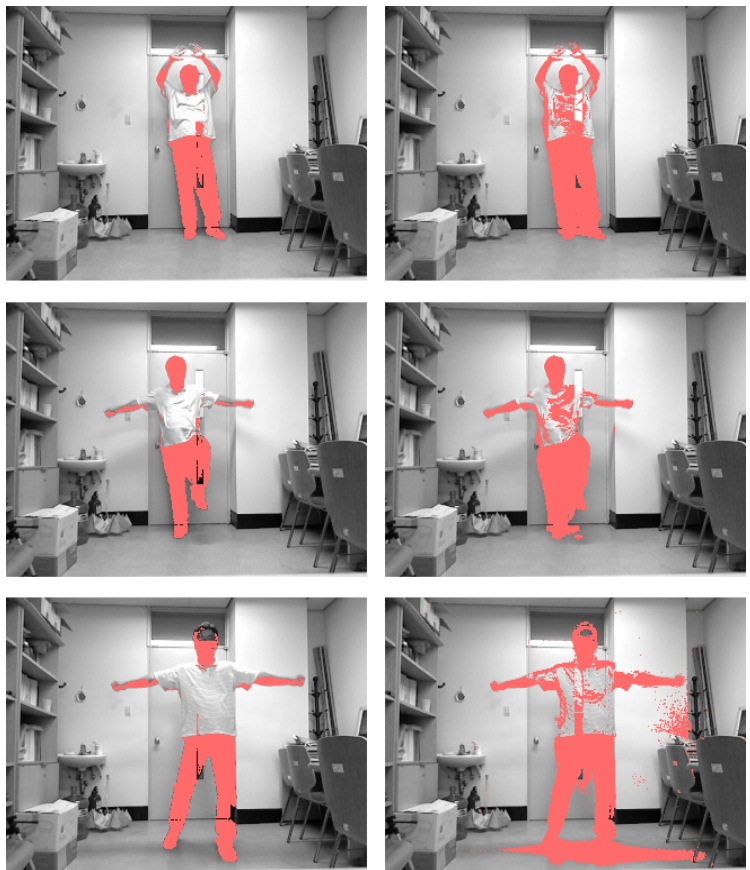

Fig. 12. Body segmentation results by the static background subtracion (left and right results are segmented by low and high threshod levels, respectively).
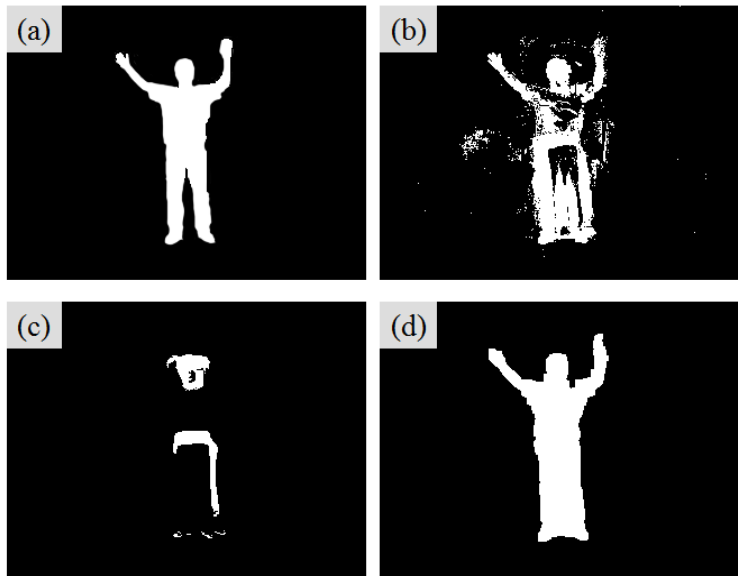

Fig. 13. A binary foreground ground truth image (a), the result of MoG background subtraction (b), the result of static background subtration (c), and the result of the proposed method (d).

For objective comparison, we used some foreground ground truth images made manually as shown in Fig. 13 (a), and compared with the results of background subtraction methods, static background and MoG background, by evaluating the mean squared error (MSE). As shown in Fig. 13 , the result of the proposed method is the most similar to the ground truth. The comparison results with some ground truth images and the average MSE comparison are shown in Fig. 14 and Table 1, respectively, where the results of the proposed method have much smaller MSEs than other methods. 


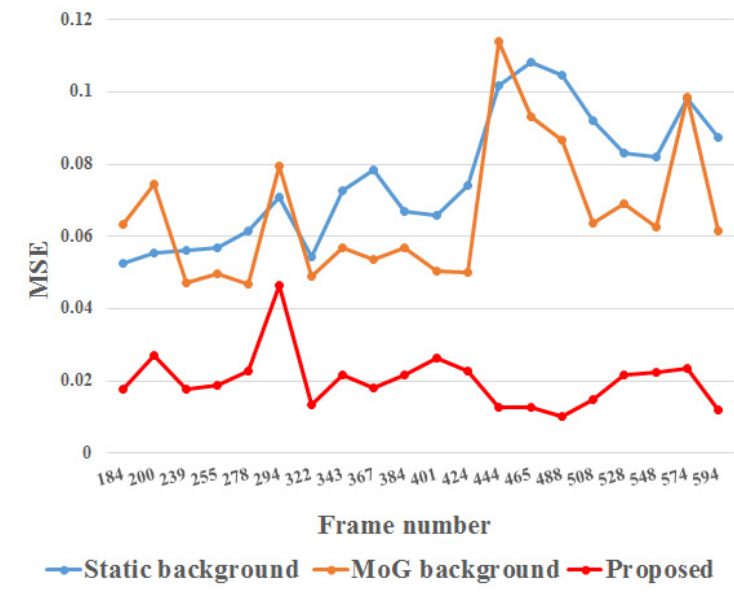

Fig. 14. MSE comparison of the proposed method and two background subtraction methods.

Table 1. Average MSE comparison with ground truth images.

\begin{tabular}{c|c|c|c}
\hline & Static background & MoG background & Proposed \\
\hline MSE & 0.0761 & 0.0663 & 0.0201 \\
\hline
\end{tabular}
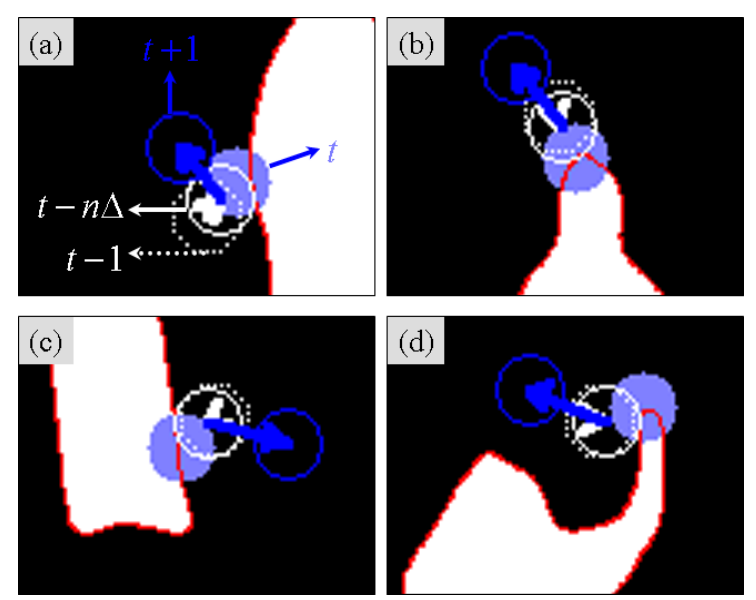

Fig. 15. Results of collision detection and response.

Fig. 15 shows that to what direction virtual object is bounced over discrete time $(t-1, t, t+1)$. Collisions with virtual objects were responded to very accurately as shown in Fig. 15, where we used $\Delta=0.1$. If the methods of $[10,13]$ and $[14]$ were used, the responding motion of the right-bottom image of Fig. 15 was in the right-top direction. However, it is not right situation when considering real physical collision. The wrong motion was corrected to the left-top direction by the proposed collision response considering intra-frame positions. That is, the proposed method more precisely reflects real physical collision phenomenon than other methods

The processing time of the proposed game, including rendering time, is about $26.79 \mathrm{~ms}$, and that is an acceptable time for real-time games.

\section{Conclusions}

In this paper, we proposed a novel framework for camera-based games by human body segmentation and collision responses. Human bodies are segmented by subtraction of gradient backgrounds and operations based on mathematical morphology, so foreground detection is not sensitive to color variances. Since the collisions between human bodies and virtual objects are estimated by the intra-frame analysis, the collision responses are very accurate. Therefore, our framework can be used for markerless camera-based games requiring high perfor-mance. Our future work includes the human activity analysis using skeletons of human bodies and tracking to improve this proposed framework.

\section{Acknowledgements}

This work was supported by a grant from the Kyung Hee University in 2012 (KHU-20120577).

\section{References}

[1] J. K. Aggarwal and M. S. Ryoo, "Human activity analysis: A review," J. ACM Comp. Surv., vol. 43, no. 3, pp. 16:1-16:43, 2011.

[2] O. Barnich and M. Van Droogenbroeck, "ViBe: A universal background subtraction algorithm for video sequences," IEEE Trans. Image Process., vol. 20, no. 6, pp. 1709-1724, Jun. 2011.

[3] D. Lee and S.G. Lee, "Vision-based finger action recognition by angle detection and contour analysis," ETRI J., vol. 33, no. 3, pp. 415-422, 2011.

[4] D. G. Zhang and Y. Zhu, "A new constructing approach for a weighted topology of wireless sensor networks based on local-world theory for the Internet of Things (IOT)," Comput. Math. Appl., vol. 64, no. 5, pp. 1044-1055, 2012.

[5] D. Zhang and Y. Liang, "A kind of novel method of service-aware computing for uncertain mobile applications," Math. Comput. Model., vol. 57, no. 3-4, pp. 344-356, 2013.

[6] D. Zhang, K. Zheng, and T. Zhang, “A novel Multicast Routing Method with Minimum Transmission for WSN of Cloud Computing Service," Soft Comput., vol. 19, no. 7, pp. 1817-1827, 2015.

[7] D. Zhang and X. Zhang, "Design and implementation of embedded un-interruptible power supply system (EUPSS) for web based mobile application," Enterprise Inf. Syst., vol. 6, no. 4, pp. 473-489, 2012.

[8] D. Lee and Y. Park, "Vision-based remote control system by motion detection and open finger counting," IEEE Trans. Consumer Electron., vol. 55, no. 4, pp. 
2308-2313, 2009.

[9] D. Lee, K. Park and Y. Park, "Collision detection and response method for markerless camera-based games using motion boundary estimation," IEEE Trans. Consumer Electron., vol. 56, no. 4, pp. 2178-2184, 2010.

[10] D. Lee and Y.J. Lee, "Framework for vision-based sensory games using motion estimation and collision responses," IEEE Trans. Consumer Electron., vol. 56, no. 3, pp. 1356-1362, 2010.

[11] Y. J. Lee and D. H. Lee, "Research on detecting face and hands for motion-based game using Web camera," in Proc. 2008 Int. Conf. Security Technology, pp. 712, 2008.

[12] D. H. Lee and Y. J. Lee, "Sensing and motion control of virtual objects for Web camera-based game," in Proc. Second Int. Conf. Future Generation Communication and Networking, pp. 28-33, 2008.

[13] D. Lee and Y. Lee, "Estimation of collision response of virtual objects to arbitrary-shaped real objects," IEICE Electron. Express, vol. 5, no. 17, pp. 678-682, 2008.

[14] D. Lee, S. G. Lee, W. M. Kim and Y. J. Lee, "Sphereto-sphere collision estimation of virtual objects to arbitrarily-shaped real objects for augmented reality," Electron. Lett., vol. 46, no. 13, pp. 915-916, 2010.

[15] Z. Tang and Z. Miao. "Fast background subtraction and shadow elimination using improved Gaussian mixture model," In IEEE Workshop on Haptic Audio Visual Environments and the Applications, 2007, pp. 541-544.

[16] Z. Zivkovic and F. Heijden, "Efficient adaptive density estimation per image pixel for the task of background subtraction," Patt. Recogn. Lett., vol. 27, no. 7, pp. 773-780, 2006.

[17] J. Park and J. Yi, "Gesture recognition based interactive boxing game," Int. J. Inf. Technol., vol. 12, no. 7, pp. 36-44, 2006.

[18] O. Oda, L. J. Lister. S. White S. Feiner, "Developing an augmented reality racing game," in Proc. Int. Conf. Intelligent Technologies for Interactive Environment, 2008.

[19] L.T. Paolis, G. Aloisio and M. Pulimeno, "A simulation of billiards game based on marker detection," in Proc. Int. Conf. Advances in Computer-Human Interactions, pp. 148-151, 2009.

[20] M. Rohs, "Marker-based embodied interaction for handheld augmented reality games," J. Virt. Real. Broadc., vol. 4, no. 5, 2007.

[21] A. Kolb, E. Barth, and R. Koch, "ToF-Sensors: New Dimensions for Realism and Interactivity," in Proc. IEEE Conf. Computer Vision Pattern Recognition, 2008.

[22] T. Leyvand, C. Meekhof, Y.C. Wei, J. Sun and B. Guo, "Kinect identify: technology and experience," Computer, vol. 44, no. 4, pp. 94-96, 2011.

[23] J. Zhu, L. Wang, R. Yang, and J. Davis, "Fusion of time-of flight depth and stereo for high accuracy depth maps," in Proc. IEEE Conf. Computer Vision Pattern Recognition, pp. 1-8, 2008.

[24] R. Crabb, C. Tracey, A. Puranik and J. Davis, "Realtime foreground segmentation via range and color imaging," in Proc. IEEE CS Conf. Computer Vision and Pattern Recognition Worksh., pp. 1-5, 2008.

[25] E. Parvizi and Q.M.J. Wu, "Multiple object tracking based on adaptive depth segmentation," in Proc. Canadian Conf. Computer and Robot Vision, pp. 273-277, 2008.

[26] T. Gonzalez-Scanchez and D. Puig, "Real-time body gesture recognition using depth camera," Electr. Lett., vol. 47, no. 12, pp. 697-698, 2011.

[27] D. G. Zhang and X. J. Kang, "A novel image denoising method based on spherical coordinates system," EURASIP J. Adv. Signal Process., vol. 1, p. 110, 2012.

[28] D. Zhang, G. Li, and X. Zheng, "An energy-balanced routing method based on forward-aware factor for wireless sensor network," IEEE Trans. Ind. Informat., vol. 10, no. 1, pp. 766-773, Feb. 2014.

[29] D. Zhang, X. Wang, X. Song, and D. Zhao, “A novel approach to mapped correlation of ID for RFID anticollision," IEEE Trans. Serv. Comput., vol. 7, no. 4, pp. 741-748, Oct.-Dec. 2014

[30] D. G. Zhang, "A new approach and system for attentive mobile learning based on seamless migration," Appl. Intell., vol. 36, no. 1, pp. 75-89, 2012.

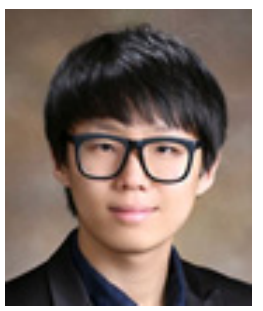

Jun-Geon Kim He received two B.S. degrees in Electronics \& Radio engineering and computer engineering and is currently pursuing an M.S. degree in Electronics \& Radio engineering at Kyung Hee University. His research interests include computer vision, pattern recognition, image processing, Electrical Impedance Tomography (EIT), 3D reconstruction and frame rate up-conversion.

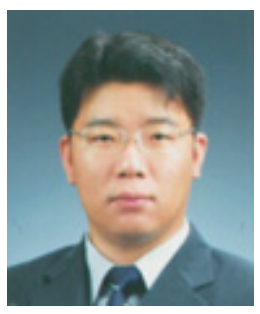

Daeho Lee He received the M.S. and Ph.D. degrees in Electronics Engineering from Kyung Hee University, Seoul, Korea, in 2001 and 2005, respectively. He has been an Associate Professor in the Humanitas College at Kyung Hee University, Korea, since 2005. His research interests include computer vision, pattern recognition, image processing, computer games, ITS (intelligent transportation system), HCI (human computer interaction), EIT (electrical impedance tomography) analysis, image fusion, and digital signal processing. 